\title{
The compression behaviors of zirconium from the first - principle calculations
}

\author{
Lin Huang $^{a}$, Xiao-Li Yuan ${ }^{a, b}$, Shou-Xin Cui ${ }^{a}$, and Dong-Qing Wei ${ }^{a, c, *}$ \\ a College of Life Science and Biotechnology and Research Center Astronautics, Shang- \\ hai Jiaotong University, Shanghai 200240, China \\ ${ }^{b}$ College of Physical Science and Technology, Sichuan University, Chengdu, 610064, \\ China \\ ${ }^{c}$ State Key Laboratory of Explosion Science and Technology, Beijing Institute of \\ Technology, Beijing 100081, China
}

Received 27 August 2012; Accepted (in revised version) 29 September 2012

Published Online 28 June 2013

\begin{abstract}
Investigation into the structural, elastic and electronic properties for pure zirconium $(\mathrm{Zr})$ crystal had been conducted by the first-principles pseudopotential method based on density functional theory. Both methods, local density approximation (LDA) and generalized gradient approximation (GGA), had been applied on the geometrical optimization of pure $\mathrm{Zr}$ to address the difference between two methods and their applicabilities. The result elucidated LDA could match the experimental data better, compared with method GGA. What's more, the structural properties under pressure had been stimulated and analyzed, showing crystal lattice parameters and crystalline volume change nonlinearly within the external pressure. In contrast, the single point energy of $\mathrm{Zr}$ showed a great linear correlation with the changing pressure. The elastic constants of the pure $\mathrm{Zr}$ were calculated, proving that $\mathrm{Zr}$ would acquire excellent ductibility and mechanical stability under pressure. In addition, the optical properties of zirconium under different pressures were analyzed. The adsorbing coefficient increased with the increasing pressure.
\end{abstract}

PACS: 31.15.es, 62.20.de, 62.20.D-

Key words: zirconium, first principles, mechanic properties, optical properties

\section{Introduction}

The key components of the space craft were mostly made by high hardness steel and sometimes dopped with titanium alloy. However, near over 40 spacial exposure experiments showed us these traditional materials have no means to meet the need for the

\footnotetext{
${ }^{*}$ Corresponding author. Email address: dqwei@sjtu.edu.cn (D. -Q. Wei)
} 
space craft in rigorous space condition, which caused people to find more light kind of materials, and metal $\mathrm{Zr}$ and its alloy might be thought as more suitable for aerospace materials, since it enjoys uncompetitive aerospace applicability . First, zirconium's excellent resistance to corrosion and low neutron-capture cross-section make it suitable for working in the space with extremely severe corrosion such as energetic particle radiation [1]. The melting spot of titanium alloy is about $1600{ }^{\circ} \mathrm{C}$, however, it is higher than $1800{ }^{\circ} \mathrm{C}$ for zirconium alloy. Thanks to its great heat resistant ability, $\mathrm{Zr}$ and its oxide have been popular applied in the production and design of aerospace craft [2-4]. Second, to achieve the real space application of the moving parts from the space crafts, the key is to achieve the strengthening-toughening and lightweight of the space materials. The strength of zirconium alloy when dopped with a small amount of $\mathrm{Sn}$ and $\mathrm{Nb}$, can reach over 900 MPa. Besides, the ductility of pure zirconium is extremely high with extensibility as $35 \%$. So the zirconium alloys system had thus powerful strengthening-toughening potential applications. Simultaneously, the density of pure zirconium is lower than steel by $16 \%$, it can be easily alloyed with some light metals. Many theoretical works had been conducted for pure $\mathrm{Zr}$ and $\mathrm{Zr}$-based alloys. Jakse et al. investigated the local structure and its change during its undercooling by means of the first principle molecular dynamics simulations. Furthermore, the research shows that for $\mathrm{Zr}$, an increasing degree of a body center cubic (bcc) type ordering upon undercooling occurs, in relation to the structure of the high temperature solid phase [5]. Hui et al. studied that the energies, pair correlation functions, structural factors and distribution of bond pairs during the liquid-solid transition of $\mathrm{Al}$ and $\mathrm{Zr}$ by ab-initio molecular dynamics simulation. Liquid $\mathrm{Zr}$ transforms to thermodynamic stable bcc phase and glassy structure around $1200 \mathrm{~K}$, when cooled at average rate of $4.3 \times 10^{13}$ and $2.0 \times 10^{14} \mathrm{~K} / \mathrm{s}$ respectively. And it is also shown that the structures of liquid and glassy $\mathrm{Zr}$ are mainly composed of icosahedral and bcc type of short range orders [6]. The composition dependence of the structural and dynamical properties of $\mathrm{Zr}-\mathrm{Ni}$ alloys had also been studied by molecular dynamics simulations [7]. What's more, our group also focused on the study of zirconium binary alloy, especially the structural properties of $\mathrm{Zr}_{2} \mathrm{Al}$ under pressure [8]. Kawamoto et al. [9] investigated the scaling trends of band offsets at model $\mathrm{Zr}$-Si interfaces, and found that the band gap and band offset decreased as the concentration of $\mathrm{Zr}$ increased. As for ternary alloys, the glassy forming stability of $\mathrm{Cu}_{46} \mathrm{Zr}_{46} \mathrm{~A}_{18}$ and $\mathrm{Fe}_{78} \mathrm{M}_{9} \mathrm{~B}_{13}(\mathrm{M}=\mathrm{Nb}, \mathrm{Si}, \mathrm{Zr})$ were investigated with ab-intio method $[10,11]$. The former one is mainly based on correlation function, Warren-Cowley parameters, as well as topology short program, to describe its glassy structure. Zr-based alloys had been investigated deeply by experimental and theoretical method. In contrast, the latter one compared the structural and dynamical properties of $\mathrm{Fe}_{78} \mathrm{Nb}_{9} \mathrm{~B}_{13} \quad \mathrm{Fe}_{78} \mathrm{Z}_{9} \mathrm{~B}_{13}$ and how the properties influence their glass forming ability, mainly based on the melting structure of $\mathrm{Fe}_{78} \mathrm{Si}_{9} \mathrm{~B}_{13}$. However, to the best of our knowledge, so far, few experimental or theoretical results had been reported for pure $\mathrm{Zr}$ metal. Under such motivation, we attempt to give a detailed theoretical study on structural, elastic, electronic and thermal properties by using first-principles plane-wave method.

In this paper, we firstly investigated $\mathrm{Zr}$ within 0-50 GPa using two exchange corre- 
lation function, GGA and LDA, based on the density function theory. Then the calculation of its elastic parameters was used to analyse its stability. What's more, through our calculation, what is also addressed in the paper is the relationship between its structural, elastic, electronic and thermal properties under extreme circumstance and pressure. Such study is not only important for pure $\mathrm{Zr}$ crystal but also valuable for us to understand the Zr-based alloys.

\section{Computational details}

We are eager to know the detailed information about $\mathrm{Zr}$ under extreme space circumstance, however, testing of the related properties under high pressure is not easy. The calculation in this paper was based on the density functional theory. At first, we cannot create so high pressure field in lab. Secondly, generally speaking, perfect result cannot be obtained easily by experiment. Thus, computer is considered to be applied to simulate the similar experiment.

Pure $\mathrm{Zr}$ is flexible silver grey metal, which is high temperature resistant with melting spot higher than $1800{ }^{\circ} \mathrm{C}$. Under normal temperature and pressure, $\mathrm{Zr}$ is quite stable, and will not react to water, oxygen, acid and alkali, with only a few exceptions, like hydrofluoric acid and aqua regia. It's space group number is $194 \mathrm{P} 63 / \mathrm{MMC}$, with hexagonal lattice structure.

The central idea of DFT is using particle density to describe the physical properties of ground states of the solid instead of the traditional quantum theory which considers wave function as the basic parameter of the system. By doing this, complicated multidimensional problems can be transformed to simple 3-dimensional ones. This theory makes incomparable contributions to the improvement of quantum computational method and the great enhance of its accuracy. All the calculations in this paper were conducted with CASTEP module in Materials Studio, which was a very important software for simulation and material design. With this, we could easily optimize the structure and conduct complex dynamics simulation. During the calculation, Perdew-BurkeErnzerhof (PBE) [12] and Cerperley-Alder(CA) were chosen to describe the exchange energy among electrons. In geometry optimization, Broyden-Fletcher-Goldfarb-Shanno (BFGS) was adopted. At the same time, the k-point was set to be $15 \times 15 \times 15$ in order to sample in the Brillouin zone, and $250.0 \mathrm{eV}$ as the cut-off energy. Before further calculation, the properties under higher cut-off energy and more k-points set had been tested, and the results did not show much difference, which meant, the parameters we chose could satisfy the accuracy demand. The crystal parameters $a=b=3.2313 \AA, c=5.1477 \AA$, $\alpha=\beta=90^{\circ}, \gamma=120^{\circ}$ obtained by experiment [13] were used to build the starting configuration of $\mathrm{Zr}$. 


\section{Results and discussion}

\subsection{Difference resulted by two simulation exchange correlation functions}

At first, the difference of geometry optimization under 0 GPa by using GGA and LDA is discussed, before simulating under extreme circumstance. The results are listed in Table 1 below. Compared computational results with the experimental data, the values obtained by GGA were higher than experimental results, however, the LDA method showed an opposite phenomenon.

Table 1: The crystal lattice constrants by different methods.

\begin{tabular}{ccc}
\hline $\mathrm{Zr}$ & $a / \AA$ & $c / \AA$ \\
\hline Present work(GGA) & 3.333 & 5.278 \\
Present work(LDA) & 3.121 & 5.115 \\
Exp.[13] & 3.232 & 5.147 \\
\hline
\end{tabular}

Based the errors when calculating $a, c$, errors by LDA method is $-3.43 \%-0.6252 \%$, respectively, with the corresponding values as 3.149\%2.538\% through GGA method. The error here was mainly caused by different processing methods dealing with the exchange correlation energy [14]. Raised by Khon-Sham [15], LDA could deal with electronic gas system very well, so it was better to be chosen to simulate systems without fierce change in its electronic density. As for GGA method, it chose electronic density and its gradient as the demanded exchange correlation energy. As a result, it could greatly enhance the accuracy of exchange correlation energy. When applied to light metal simulation, highly consistency compared with experiment results could be obtained [16].

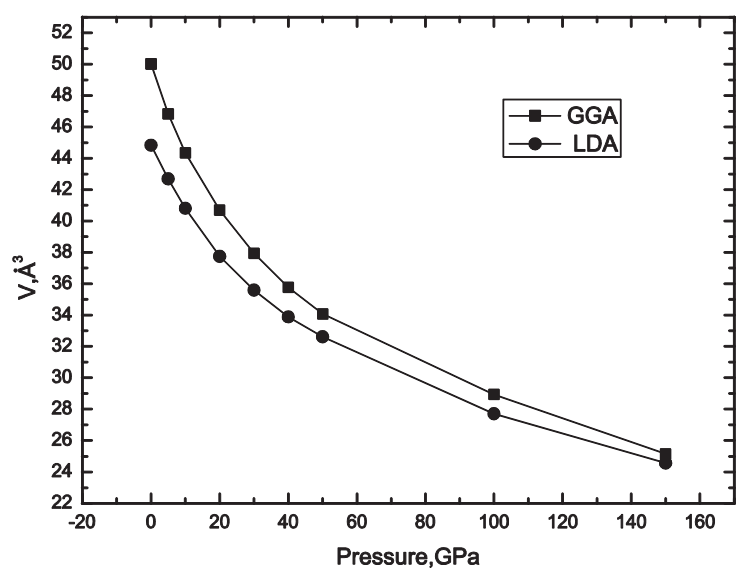

Figure 1: Volume-pressure curves of zirconium.

Then the relationship between crystalline volume and pressure changing from 0-50 GPa was also analyzed. Crystalline volume is a very important characteristic, which can 
be used to predict pressure induced phase transformation. Since when pressure increasing, the crystalline volume will keep on shrinking, which will result in probable lower single point energy than any other stable phase under normal pressure. Fig. 1 showed how the volume changes according with the changing pressure. From the figure, the volume decreases, when external pressure goes up.

Obviously, the values achieved by GGA were higher than by LDA. However, when the pressure became higher, two curves became nearer, which meant precise results would be achieved under high pressure no matter which method chosen to use. The probable reason for this phenomenon might be when the pressure got higher, the distance among molecules became shorter, at the same time, the Vander Walls force changed stronger, so that two kinds of methods were all quite suitable to simulate this system, thanks to the lack of intermolecular force in the system. What's more, theoretical analysises on transition metal elements showed an close result between LDA adopted simulation and GGA, suggesting it's suitable to simulate transition metal elements by either method [16]. However, the result obtained by LDA was closer to the experimental values, indicating theoretical computation could polish and perfect the former imcomplete experimental data.

Based on Ref. [17], there were crystal lattice stable parameters as well as Gibbs energy of 78 kinds of elements above $298.15 \mathrm{~K}$ in the SGTE (Scientific Group Thermo data Europe). Wang et al. [18] computed these pure element by pseudopotential method systematically, and compared detailedly with data from SGTE Databank, Saunders C223 Databank and Skriver E233 Databank. The crystal stability of Zr thus was proved as 3.61.

The Fig. 2 showed how the crystal parameter changed with pressure. From 0-50 GPa, compression ratio of $a, c$ calculated by GGA were $16.12 \%$ and $4.57 \%$, respectively, and $11.15 \%$ and $6.62 \%$ by LDA. Figs. 3,4 showed the compression ratio $a / a_{0}$ and $c / c_{0}$.

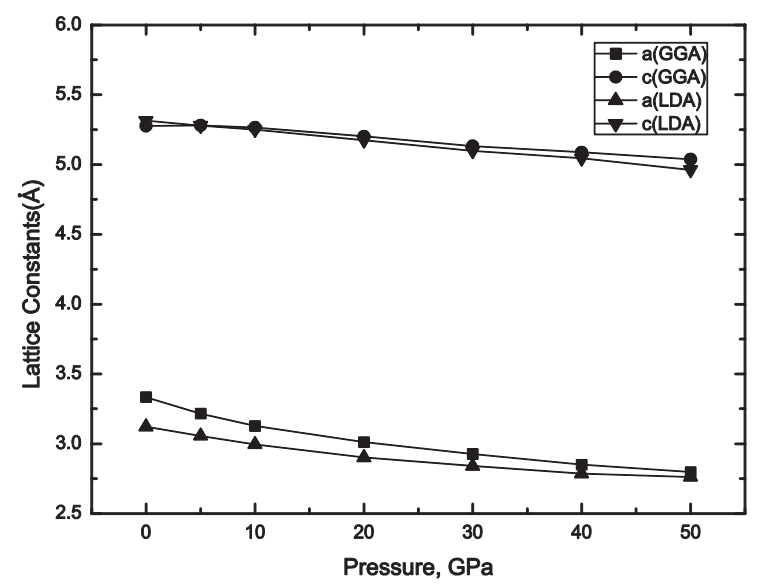

Figure 2: Relationship between cystal lattice parameters and pressure

The energy under different pressure was listed in the table below. The energy in- 
creased when the pressure increased. Based on the data in Table 2, the energy-pressure relation could be present in Fig. 5, with amazing linear correlation degree. Fixing it linear, the linear correlation degree were calculated as 0.9991 , with error $<0.0001$, which could be considered as linear. The fixing linear function followed as:

$$
E=-2.561 .4647+0.24922 P
$$

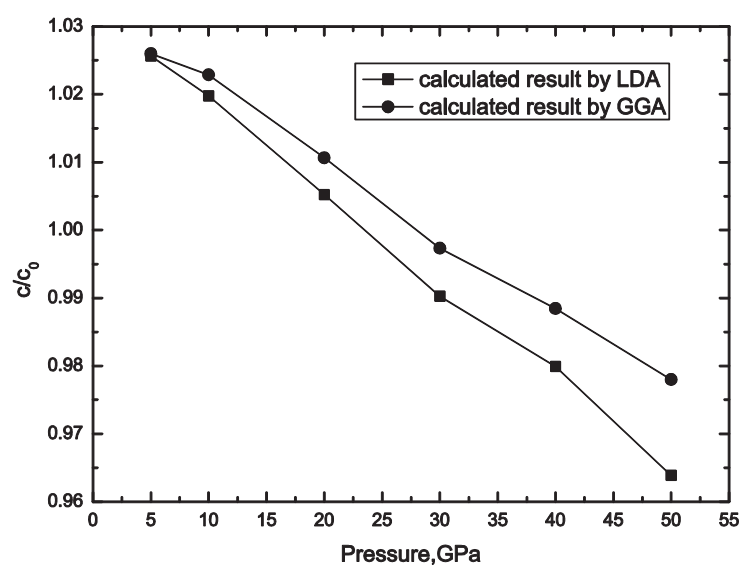

Figure 3: Compression ratio of $\mathrm{c} / \mathrm{c} 0$ with pressure.

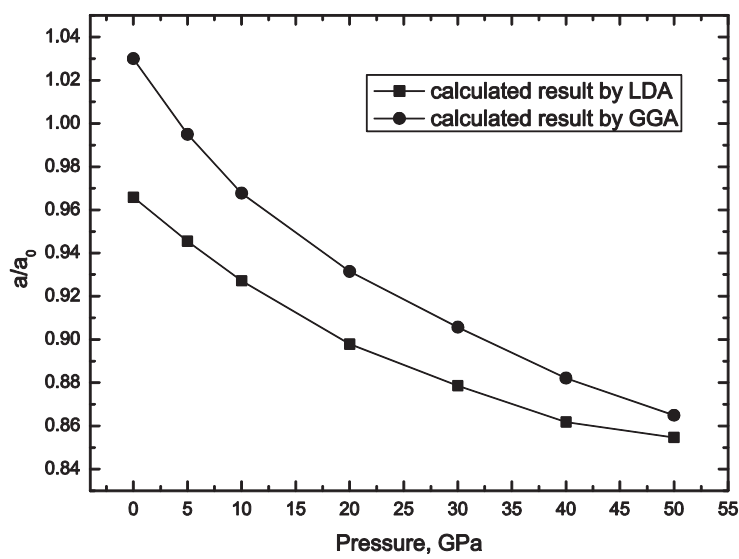

Figure 4: Compression ratio of $\mathrm{a} / \mathrm{a}_{0}$ with pressure.

Increasing the pressure would lead to the increasing of the energy of material. When the pressure reached a specific value, the energy of other phase might be lower than the

Table 2: Energy under different pressure.

\begin{tabular}{ccccccc}
\hline Pressure $/ \mathrm{GPa}$ & 0 & 5 & 10 & 20 & 30 & 40 \\
\hline Energy /eV & -2561.67 & -2560.22 & -2558.85 & -2556.29 & -2553.91 & -2551.68 \\
\hline
\end{tabular}




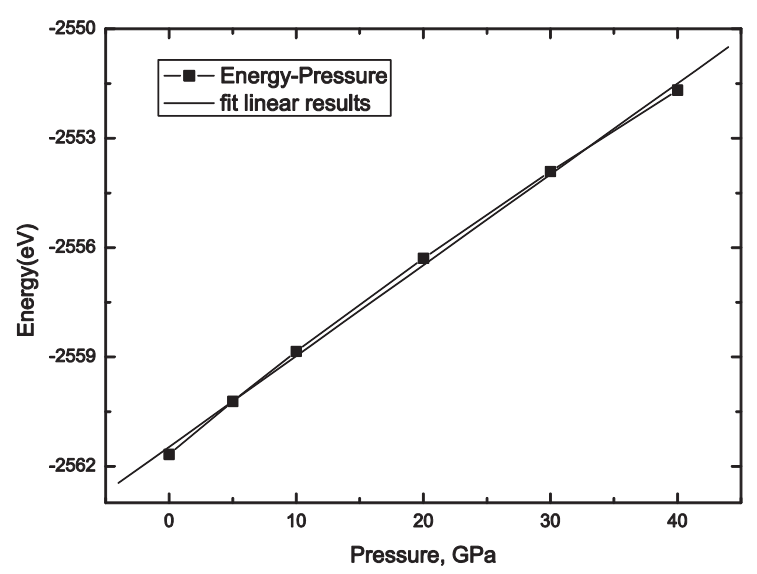

Figure 5: Single point energy under varied pressure.

existing one, which might lead to the probable phase change. System always tended to have the lowest energy, to keep itself stable. So under the specific pressure, this phase of zirconium might turn into another phase and the phase transition occured. Whether changing pressure would have real impact on the phase change of Zr still awaited study.

\subsection{The elastic properties of $\mathrm{Zr}$}

The elastic modulus determined the response of the crystal to the external forces, and played an important role in deciding the strength of the materials. When one kind of material reacted to the external force, the shape of the material would have some changes. At the same time, the total energy of the material would also have corresponding changes. By calculating the second order partial derivative of the material energy, therefore the elastic constants of the material can be obtained.

Five independent elastic parameters of the primitive cell in the hexagonal lattice are $\mathrm{C}_{11}, \mathrm{C}_{12}, \mathrm{C}_{13}, \mathrm{C}_{33}$ and $\mathrm{C}_{44}$ respectively, moreover, $\mathrm{C}_{66}$ could be derived from the independent parameters above. Thus, only 5 independent ones were used to examine its elastic properties. From Table 3, we could see that the value of elastic constants, bulk modulus and Young's modulus increased with increasing pressure. Considering hexagonal lattice structure, its mechanical stability could be derived by the following formula under isotropic pressure [19]:

$$
\hat{C}_{44}>0, \hat{C}_{11}>\left|\hat{C}_{12}\right|, \hat{C}_{33}\left(\hat{C}_{11}+\hat{C}_{12}\right)>2 \hat{C}_{13}^{2}
$$

where

$$
\begin{aligned}
& \hat{C}_{\alpha \alpha}=C_{\alpha \alpha}-P(\alpha=1,2, \cdots 6) \\
& \hat{C}_{12}=C_{12}+P \\
& \hat{C}_{13}=C_{13}+P \\
& \hat{C}_{23}=C_{23}+P
\end{aligned}
$$


Table 3: Elastic constants $\mathrm{C}_{i j}$, bulk modulus $\mathrm{B}$ and Young modulus under pressure.

\begin{tabular}{ccccc}
\hline & $0 \mathrm{GPa}$ & $20 \mathrm{GPa}$ & $40 \mathrm{GPa}$ & $50 \mathrm{GPa}$ \\
$\mathrm{C}_{11}$ & 103.36745 & 222.91675 & 302.33350 & 319.40620 \\
$\mathrm{C}_{13}$ & 48.30467 & 116.50285 & 154.41247 & 173.90800 \\
$\mathrm{C}_{33}$ & 123.99765 & 205.36380 & 289.90410 & 141.34550 \\
$\mathrm{C}_{12}$ & 44.31493 & 132.96755 & 110.98600 & 365.98195 \\
$\mathrm{C}_{44}$ & 28.54995 & 17.50110 & 17.87525 & 10.06810 \\
Bulk modulus(B) & 68.21069 & 141.71776 & 194.12640 & 277.45526 \\
Young modulus(X) & 77.73629 & 127.06186 & 218.59275 & 157.13720 \\
Young modulus(Y) & 71.70534 & 95.76593 & 175.05252 & 1186.35033 \\
Young modulus(Z) & 85.77847 & 97.02216 & 149.85078 & 3352.91205 \\
\hline
\end{tabular}

Through Table 3, conclusion could be drew like the elastic constants, Bulk Modulus and Young Modulus were increasing with increasing pressure, which was induced by volume deformation under high pressure. If this system was mechanical stable under any external force, five independent elastic constants should satisfy the well-known Born stability criteria [20]. Generally speaking, if the $C_{44}$ of a crystal was larger than 0 , it was capable of guaranteeing systematically mechanical stability. Based on this criteria, this crystal could be considered mechanical stable. Zirconium crystal had a relatively large bulk modulus, which indicated that it could resist plastic deformation.

The number of strain modules directly decided the computational complexity. By stress-strain, the complexity of computation could be greatly decreased, however, in this way the higher requirements had been raised on the capability of the first-principles computational softwares to deal with crystalline Cauchy stress tensor. The CASTEP module from Material Studios is among a few amount of softwares which could directly deal with Cauchy tensor. So, it can be easily applied to compute the second order elastic constants of a kind of material.

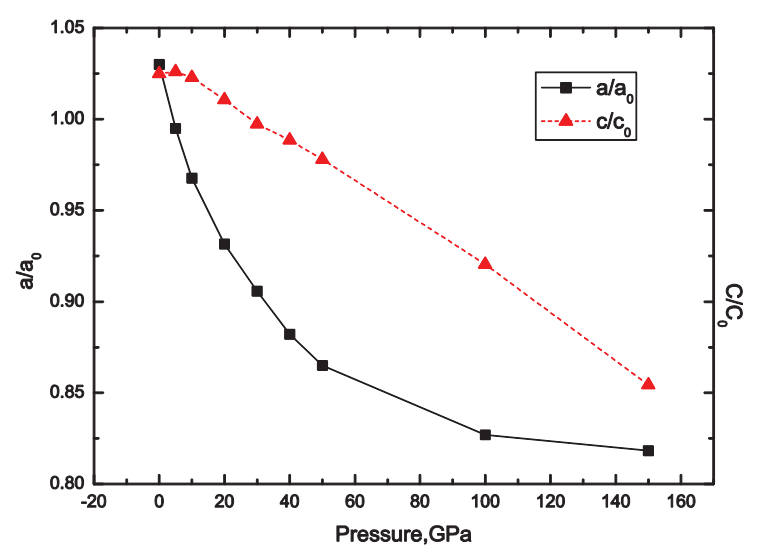

Figure 6: Compression ratio of $\mathrm{Zr}$ under increasing pressure. 
There was a very interesting phenomenon in Fig. 6 that the compression ratio along a-axis was much larger than along c-axis. It might be explained as the bond energy along c-axis was less than along a-axis. To know this problem completely, the density functional theory should be used to calculate the charge density distribution in different direction of the lattice.

\subsection{The electronic system of $\mathrm{Zr}$}

Fig. 7 showed the energy bands from 0-20 GPa. The whole wave functions gave out all energy bands, however, what drew the most attention only was the energy bands near the Fermi energy band. Calculating all energy bands was not only time consuming, but also not practical. So CASTEP just calculated finite energy bands close to the Fermi band.

The energy bands were important signature for describing the electron system of the crystal. The dashed line in the figure represented Fermi level. Above the Fermi level, it was conduction band. And below it, there was the valence band. The Fermi band of zirconium was between the conduction band and the valence band, which means it was a kind of metal. The distribution of valence band and conduction band in the whole Brillouin zone was relatively smooth, which was determined by the feature of crystal zirconium. There was no band gap between the valence band and the conduction band. The precondition to become a good conductor was the Fermi band could crossover other bands as much as possible, moreover, if the Fermi level crossed over some narrow bands, the localized state would be produced and the electrical conductivity would not deserve to be await [21]. The energy bands which the Fermi level crossed over were relatively wide in crystal zirconium, which suggested this kind of metal had an excellent conductivity.

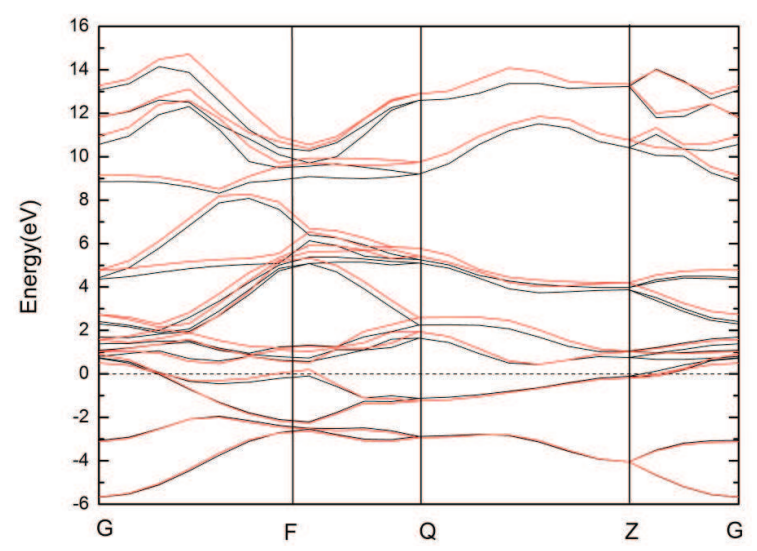

Figure 7: Energy band structures of Zr under 0GPa (black lines) and 20GPa (red lines); Dashed lines represented Fermi energy band.

The curves in different color from Fig. 7 showed no much difference in band structure, which means pressure would influence the electronic structure so little that it could not 


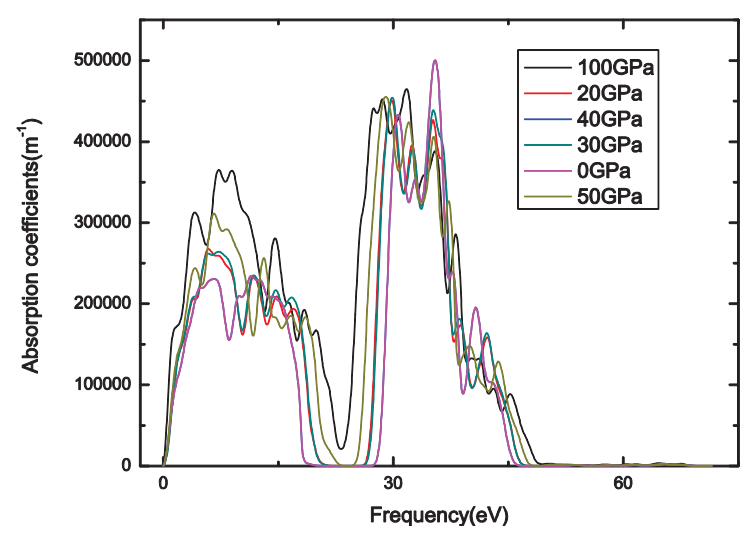

Figure 8: Absorbing coefficients under varied pressure.

be considered as an impact factor.

\subsection{Optical properties}

Generally speaking, the interaction between photon and electron might lead to the transition between occupied and unoccupied states. The optical properties were resulted by the electronic transition, which made great demands for the accuracy of the computational result about the energy band structure.

From the Fig. 8, the shapes of the absorption coefficient curves were almost similar, but the corresponding values were different. The peak value of the absorption coefficient in the basic zone was considered relevant with the different excited states. With the increasing of pressure, the absorption coefficients also increased and reached to its maximum under $100 \mathrm{GPa}$.

\section{Conclusions}

Paper here discussed several kinds of characteristics of zirconium under increasing pressure based on the density function theory. Below were the main conclusion.

1. LDA and GGA method were examined to simulate $\mathrm{Zr}$ from 0-50 GPa, and its crystal structure was also analyzed. According to the analysis in 3.1, two derived structures did not give out a same result. GGA tended to overestimate the computational results, on the contrary, LDA showed a opposite result.

2. Then the energy band structures by stimulating the electronic structure of zircon were achieved under different pressures. Based on the analysis on its band structures, Zr was indeed belong to metal., which provided strong evidence for our simulation. What's more, its electronic structure would not be influenced by pressure greatly. Even though, the peak value of energy band still kept decreasing with the increasing of pres- 
sure., which showed within a certain energy value, the numbers of energy bands would decrease, when rasing up the external pressure. Thus changing pressure could alter the energy band structure to some extent. Besides, analysing the single point energy of $\mathrm{Zr}$ and pressure would indicate there was linear relation between them.

3. As for its elastic characteristic, the obtained elastic coefficients proves the stability of $\mathrm{Zr}$.

4. Last, the optical property under different pressures were computed and analyzed. The absorbing coefficients increased as the the increase of pressure, and reached its maximum at $100 \mathrm{GPa}$.

Acknowledgments This work is supported by grants from the State Key Laboratory of Explosion Science and Technology, Beijing Institute of Technology (Grant No. KFJJ0902) the National Basic Research Program of China (Grant No. 2010CB731600), and the National Key Laboratory for Nuclear Fuel and Materials of Nuclear Power Institute of China (Grant No. STRFML-2012-06).

\section{References}

[1] D. R. Lide, Zirconium, CRC Handbook of Chemistry and Physics ( CRC Press, New York, 2007-2008).

[2] A. Stwertka, A Guide to the Elements (Oxford University Press,USA, 1996).

[3] S. M. Meier and D. K. Gupta, J. Eng. Gas Turb. Pow. 116 (1994) 250.

[4] E.R. Krebs, The History and Use of Our Earth's Chemical Elements (Greenwood, Westport, 2006).

[5] N. Jakse, O. L. Bacq, and A. Pasturel, J. Non-Cryst. Solids 353 (2007) 3684.

[6] X. D. Hui, H. Z. Fang, and E. X. Wang, Sci. China Phys., Mecha. \& Astron. 40 (2010) 1208 (in Chinese).

[7] T. Aihara Jr., Y. Kawazoe, and T. Masumoto, J. Non-Cryst. Solids 207 (1996) 875.

[8] X. L. Yuan, D. Q. Wei, X. R. Chen, et al., J. Alloy. Comp. 509 (2011) 769.

[9] A. Kawamoto, K. Cho, P. Griffin, et al., J. Appl. Phys. 90 (2001) 1333.

[10] S. P. Pan, J. Y. Qin, T. K. Gu, et al., J. Appl. Phys. 105 (2009) 13531.

[11] H. Z. Fang, X. Hui, G. L. Chen, et al., Appl. Phys. Lett. 94 (2009) 091904.

[12] J. P. Perdew, K. Burke, and M. Ernzerhof, Phys. Rev. Lett. 77 (1996) 3865.

[13] B. Olinger and J. C. Jamieson, High Temp.- High Press. 2 (1973) 513.

[14] Y. Zhang, Computational Materials Science (Beijing University of Aeronautics and Astronautics Press, Beijing, 2007) (in Chinese).

[15] W. Kohn and L. J.Sham, Phys. Rev. Lett. 140 (1965) 1133.

[16] J. H. Tao and W. M. Chen, Matt. Rev. 23 (2009) 77.(in Chinese)

[17] A. T. Dinsdale, Calphad 15 (1991) 317.

[18] Y. Wang, S. Curtarolo, and C. Jiang, Calphad Compt. Coupl. of Phas. Diag. \& Therm 28 (2004) 79.

[19] G. V. Sinko and N. A. Smirnov, J. Phys.: Conddens. Matt. 14 (2002) 6989.

[20] M. Born, Proc. Cambridge Philos. Soc. 36 (1940) 160.

[21] K. A.Gschneidner, Sol. Stat. Phys. 16 (1964) 275. (in Chinese) 